\title{
Research on CAD Key Technology for Crystal Lamps with Body Shape Distribution
}

\author{
Yanxia LIANG ${ }^{1}$ \\ ${ }^{1}$ College of Mechanical and Electrical Engineering, \\ Jiangsu Normal University, \\ Xuzhou, Jiangsu 221116, China, \\ Lyx_xznu@sina.com
}

\begin{abstract}
With colorful and changing light effect, crystal lamp has become the first choice for the indoor lighting facilities. Crystal lamps have many identical or similar elements in structure, so using CAD technology can assist the designers for the design and modeling of crystal lamps rapidly. In this paper, the distribution of crystal lamp is divided into two types of combined closed surface and a single closed surface. For these two types, the method of determining the distribution point of the main crystal is given, and the pseudo code is provided. The VB language is applied to develop the parameter modeling plug-in applicable to the auxiliary design of crystal lamps based on Rhinoceros platform, and the designers use the plug-in to automatically complete the 3D model creation of crystal lamps by selecting parameters and assigning values to the variables, and thus achieving the parameter modeling and improving the design efficiency.
\end{abstract}

Keywords-- body shape Distribution; crystal lamps; computer aided design; combined closed surface frame; single closed frame

\section{I .INTRODUCTION}

Decorative lighting is an important part of interior environment design. It is believed that the lighting design is the focus of interior design in the future and gorgeous crystal lamp will surely attract the attention of consumers and will be their first choice for the interior decoration[1].

With the development of the crystal lamp industry, the lamp producers and suppliers are required to shorten the product cycle and improve their design capabilities. But in recent modeling process for crystal lamp, most designers have to complete the work through the complex and tedious process manually. Once the modeling is complete, the modification process is quite cumbersome. If some mistakes have been found, it will greatly increase the designers' burden. Nowadays, a great creative space for crystal lamp design has been provided to designers because of the digital techniques development. It has to be an inevitable trend to use a CAD technology for crystal lamp modeling and design. Rhinoceros software is a very convenient and interactive 3D modeling software, and it is easy to master. It also provides a development port. In this study, a plug-in has been developed for crystal lamp aided design which used VB language, based

Project supported by the Natural Science Foundation of the Jiangsu Higher Education Institutions of China ( Grant No. 15KJD460005) and the Jiangsu Normal University Natural Science Foundation (Grant No.10XLA17) .

\author{
Guangguang DONG ${ }^{2}$ \\ ${ }^{2}$ ZheJiang RongChuang information and Industry company, \\ LTD. \\ Hangzhou, Zhejiang 310013, China \\ 327901266@qq.com
}

on the Rhinoceros.

According to the structure characteristics, crystal lamps can be divided into three different basic types, that is, linear distribution, planar distribution and body shape distribution. As a body shape distribution, all crystal units distribute along closed surface and the internal, as shown in Fig.1[2,3,4].
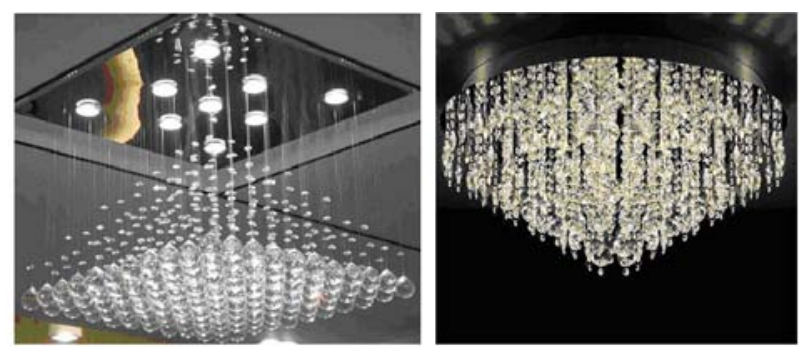

Fig.1 body shape distributions of crystal lamp

Based on the design idea of the designer and the characteristics of CAD system, this paper presents the design process of body shape crystal lamp, as shown in Fig.2.

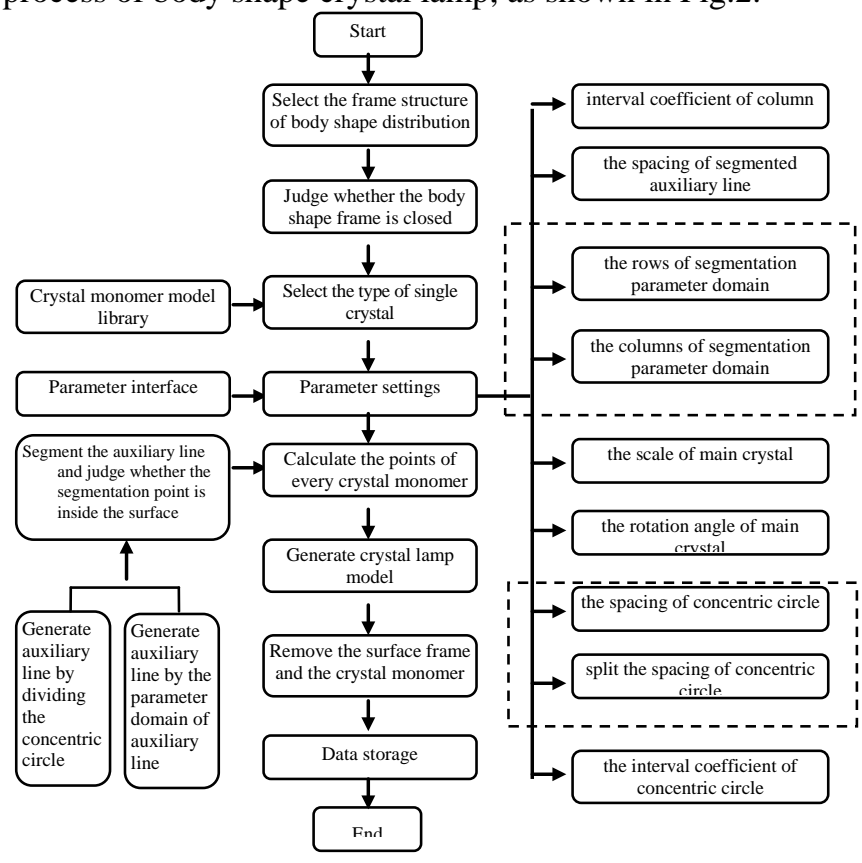

Fig.2 design flow of crystal lamps with body shape distribution framework 


\section{II .CAD KEY TECHNOLOGY OF CRYSTAL LAMP WITH BODY SHAPE DISTRIBUTION}

According to the frame structure feature of crystal lamp, the body shape distribution of crystal lamp is divided into two types of combined closed surface and a single closed surface in this study[5]. In view of these two kinds of frame structure crystal lamps, the design of CAD key technologies are as follows:

\section{A. Method for determining the distribution point of the main crystal}

As a rapid design method for ordinary body shape framework, it is the key point that determining the main crystal distribution points during the design process[5]. There are two implied direction parameters in NURBS (Non-Uniform Rational B-Surface), in which the control points of the parameter domain can be seen as designed point of our crystal lamp, as shown in Fig.3.

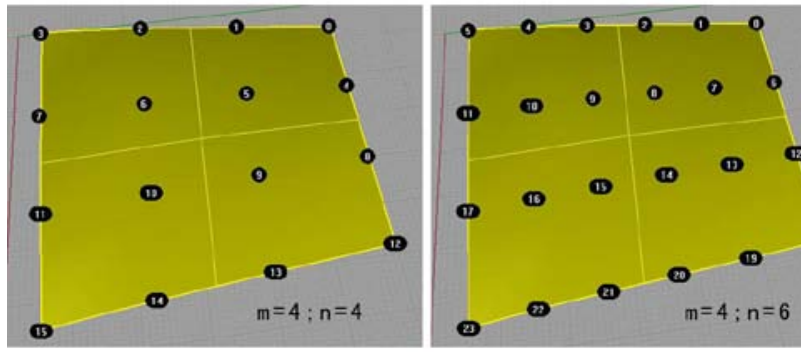

Fig.3 control points of the ordinary framework surface domain

In another way, guidelines can be generated on Z-axis direction firstly, and then, intersections of guidelines and ordinary framework surface can be got by calculation. In general, these guidelines always through break points which partition a series of concentric circles in the surface frame work. In addition to this method, we can make an auxiliary plane with Boundingbox() function first. Control points in the parameter domain of the auxiliary plane can be calculated and saved. Then, guidelines can be generated through these saved control point. The distribution forms of auxiliary lines are showed in Fig. 4.
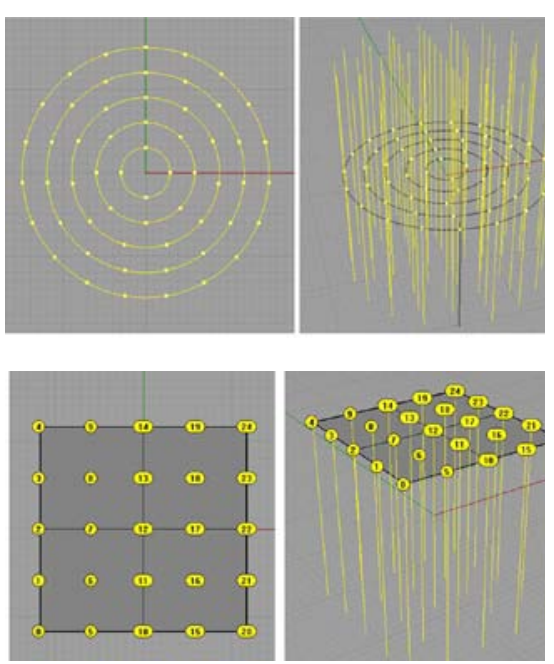

Fig.4 methods for guideline generation
Through subdivision surface parameter domain, save plane control points, and then calculate the minimum distance of adjacent control points in the direction of $U$ and $V$, takes two relatively small value of the minimum value, as all the minimum distance between adjacent control points, and then calculate the scaling. Its pseudo code is as follows:

Select the plane frame;

Calculate the parameter domain of surface $\mathrm{U}, \mathrm{V}$ direction;

Subdivide the parameter domain of surface $\mathrm{U}, \mathrm{V}$ direction;

For $\mathrm{i}=0$ to $\mathrm{m}-1(\mathrm{~m}$ - the $\mathrm{U}$ partition number of surface parameter domain);

For $\mathrm{j}=0$ to $\mathrm{n}-1(\mathrm{n}$ - the $\mathrm{V}$ partition number of surface parameter domain);

Transform the two-dimensional array to one-dimensional array;

Output all the control points of the surface;

Next;

Next

\section{B. Key technology of crystal lighting design for two kinds of} frame structures

\section{1 ) the frame type of combined closed surface}

For the frame type of combined closed surface, the distribution points of the main crystals can be determined through the above - mentioned method, and we need to to judge whether the segmentation points are in the surface and internal of the combined closed surface, only those in the surface and internal of the combined closed surface can be as the distribution points of the main crystals.

\section{2 ) the frame type of single closed surface}

For the frame type of single closed surface, the function CurveSurfaceIntersection () can be used to solve the intersection of the line and surface. The method which determine the distribution points of the main crystals can be divided into two kinds: the first is the above - mentioned method; the second is through the generation of secondary auxiliary lines, divided the secondary auxiliary lines. The specific methods are as follows:

a)Generate the auxiliary line using the method introduced in II A .

b) Solve the intersection point between the auxiliary

line and the single closed surface, and judge the number of intersection point.

c) If the intersection number is greater than one, the

secondary auxiliary line is generated by the first intersection and the last one, as shown in Fig.5.

d) Split the secondary auxiliary lines, and save the segmentation points.

e) Judge whether the segmentation point is in the surface or the inside of the single closed surface, if is, then it will be as the main crystal distribution point.

The advantage of the above method is to be able to extract more control points on the closed surface, and the user 
can control the main crystal types which located on the surface distribution points and the internal distribution points.

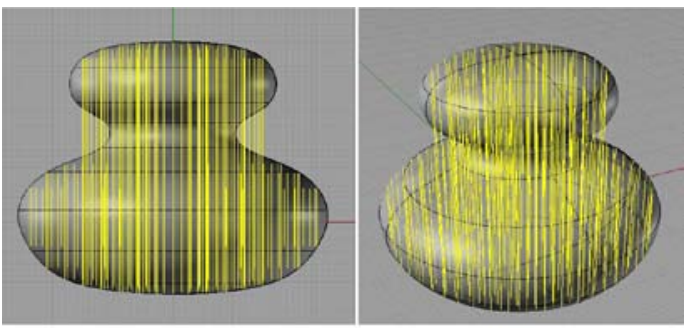

Fig.5 generate secondary auxiliary lines

Once the main crystal distribution points are determined, the types of main crystal can be one or two. The method which distribute one main crystal is to copy the main crystal to the distribution point. The method of distributing two main crystals is as follows:

The first situation is with a single concentric circle as a unit, the main crystal type is same which located in the same concentric circle. The user determines the number of the two main crystals between the intervals of concentric circles.

The second situation is with the row of parameter domain of the subdivision auxiliary surface as a unit, the main crystal type is same which located in the same row. The user determines the interval rows which between the two main crystals.

\section{DESIGN PRACTICE OF THE BODY SHAPE DISTRIBUTION CRYSTAL LAMP}

The number of the main crystals is very much on body shape distribution frame, and its styling is full, so it can achieve good effect of light reflection. Based on this, there is no need to distribute small crystal on the crystal rope. The design key steps are given as follows:

\section{A. the frame type of combined closed surface}

Based on Rhino software, designer create the frame structure of combined closed surface and the line hanged surface by hand, as shown in Fig.6[6,7]. Then determine the distribution points of the main crystals using the above-mentioned method, and operate the developed Rhino plug-in. In the dialog box of the design interface, designer can choose crystal kinds of monomer, load the model of crystal monomer, input the related parameters, then the 3D model of crystal lamp will be generated automatically by the design system. Then delete the frame line and the loaded crystal model, the result of the design is shown in Fig.7. The crystal monomer is distributed by matrix, as shown in Fig.8.

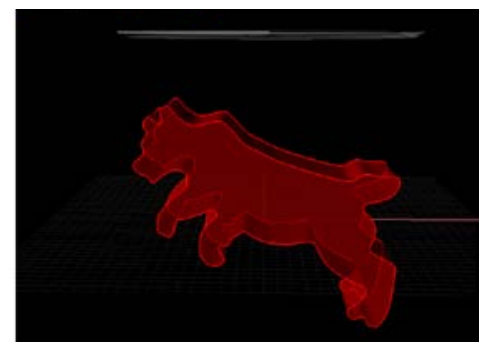

Fig.6 the frame structure of combined closed surface

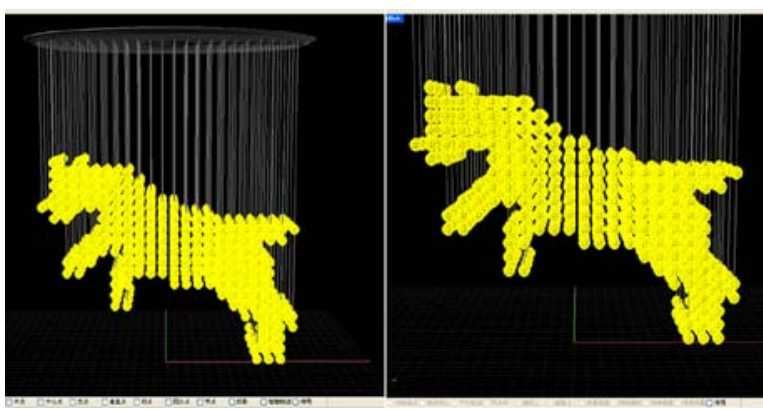

Fig.7 the 3D model of crystal lamp

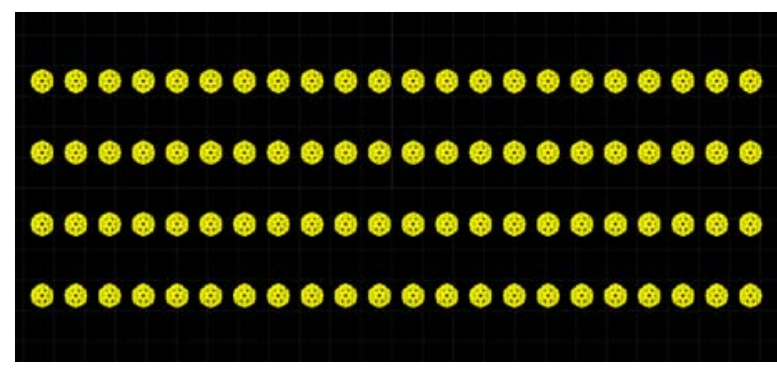

Fig.8 The matrix-distributed crystal monomer

\section{$B$. the frame type of single closed surface}

The model generation process of the single closed surface frame type is similar with the above combined closed surface frame type, the only difference is that the drawing of the body frame structure and the distribution forms of crystal monomers are different, its generation process as shown in Fig.9 to Fig.11.

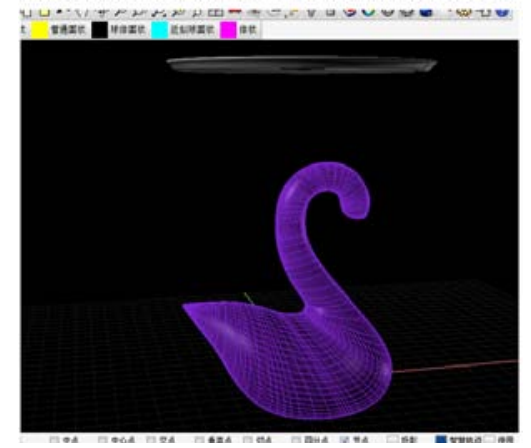

Fig.9 the frame structure of single closed surface
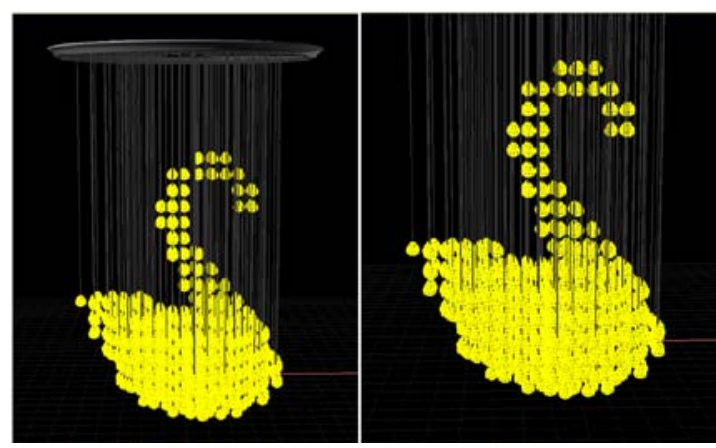

Fig.10 the 3D model of crystal lamp 


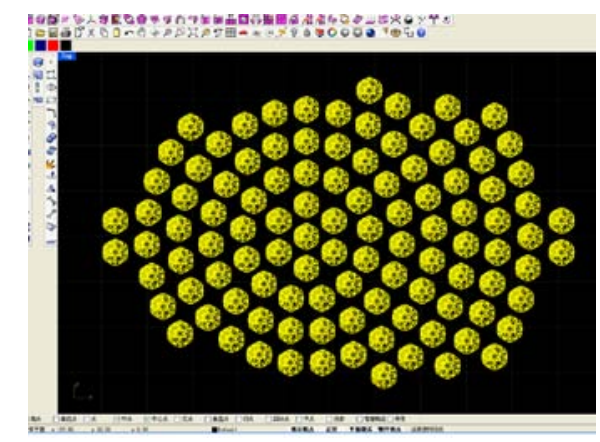

Fig.11 The concentric circle distributed crystal monomer

\section{CONCLUSION}

This research analyzes the structural characteristics of the crystal lamps, and they are divided into liner distribution, plane structure and body shape structure. For the crystal lamps of body shape distribution, a parameter modeling plug-in applicable to the auxiliary design of crystal lamps is developed based on Rhinoceros platform, which can free the designers from the heavy modeling process, through parameter selection and variable assignment, the design plug-in automatically completes the creation of crystal lamp 3D model, achieves parameter modeling and improves efficiency. Besides, it is of convenient modification and can determine the position of crystal monomer more rapidly and accurately, thus omitting the complicated calculation process of designers.

Due to the complicated and changing morphology of crystal lamps, the parameter plug in for the auxiliary design of crustal lamps developed in this research cannot be applicable to the crystal lamps of all modeling. In the next, deep research will be conducted on the calculation method of size and zooming proportion of crystal monomers.

\section{REFERENCES}

[1] Michael J Ray. Crytal Lamps Used In Home Design[EB/OL]. http://ezinearticles.com/?Crytal-Lamps-Used-In-Home-Design\&id=51498 68. 2010-08-07/ 2010-10-20.

[2] Peter K. H. Huang. Crystal lamp bulb[P]. U.S: Des.426,901. 2000-7-20.

[3] Andrew Schonbek.. Crystal ornament for lamp[P]. U.S: US D503,011 S . 2005-3-15.

[4] Michael R. Kling et al. Spiral facetted crystal decor lamp[P]. U.S: US D501,262S. 2005-1-25.

[5] Su Huan, Liu Xiaojian. Study on the rapid design techniques of crystal lighting[C].2010 IEEE 11th International Conference on Computer Aided Industrial Design and Conceptual Design, CAID and CD'2010.YiWu,Zhejiang:2010: 797-799.

[6] Pan Li, Jiang Wen. Tiffany shade modeling system based on Rhino development $[\mathrm{J}]$. Computer engineering and design, 2007.28 (18): 4426-4428.

[7] Yang Huixian, Yang Sui, Wang Zihan. The skills of Rhino development based on VB platform [J]. computer study, 2004.6 (3): 40-41. 